\title{
Image Guidance for Spinal Facet Injections Using Tracked Ultrasound
}

\author{
John Moore ${ }^{1}$, Colin Clarke ${ }^{2}$, Daniel Bainbridge ${ }^{2,3}$, Chris Wedlake $^{1}$, \\ Andrew Wiles ${ }^{1,4}$, Danielle Pace ${ }^{1,5}$, and Terry Peters ${ }^{1,4,5}$ \\ 1 Imaging Research Laboratory, Robarts Research Institute, London, Canada \\ jmoore@imaging.robarts.ca \\ 2 Division of Anesthesia, The University of Western Ontario, Canada \\ 3 Canadian Surgical Technologies and Advanced Robotics (CSTAR), Canada \\ 4 Biomedical Engineering Graduate Program, The University of Western Ontario, \\ Canada \\ 5 Department of Medical Imaging, Department of Medical Biophysics, \\ The University of Western Ontario, Canada
}

\begin{abstract}
Anesthetic nerve blocks are a common therapy performed in hospitals around the world to alleviate acute and chronic pain. Tracking systems have shown considerable promise in other forms of therapy, but little has been done to apply this technology in the field of anesthesia. We are developing a guidance system for combining tracked needles with non-invasive ultrasound (US) and patient-specific geometric models. In experiments with phantoms two augmented reality (AR) guidance systems were compared to the exclusive use of US for lumbar facet injection therapy. Anesthetists and anesthesia residents were able to place needles within $0.57 \mathrm{~mm}$ of the intended targets using our AR systems compared to $5.77 \mathrm{~mm}$ using US alone. A preliminary cadaver study demonstrated the system was able to accurately place radio opaque dye on targets. The combination of real time US with tracked tools and AR guidance has the potential to replace $\mathrm{CT}$ and fluoroscopic guidance, thus reducing radiation dose to patients and clinicians, as well as reducing health care costs.
\end{abstract}

\section{Introduction}

A wide variety of pain conditions are treated with needle injection therapies. Selective nerve blocks require careful placement of the injection needle, both to ensure effective therapy delivery and to avoid damaging neighboring tissues. The facet joint is a source of chronic pain for between $15 \%$ to $45 \%$ of patients with chronic lower back pain[1. Lumbar facet joint injections are a good example of a particularly challenging therapy; the small, narrow channel between vertebrae, the oblique entry angle, relatively deep location and proximity to nerve tissue can make accurate treatment delivery quite difficult.

Most facet injections are performed using fluoroscopy or CT guidance, which involves delivering radiation dose to the patient and health care providers. Recently, 
a number of groups have been developing guidance techniques with the goal of reducing or eliminating this radiation dose while either maintaining or improving patient safety. For example, Proschek et al [2] have developed a guidance system that uses a laser projection system to limit the radiation dose required for needle delivery. Magnetic tracking systems (MTS) have shown promise for improving the guidance of a wide variety of interventional procedures [3]. Tracking technology is relatively inexpensive compared to 3D US systems or fluoroscopy, making it a viable option for any clinical setting. Bruners et al have demonstrated a system that combines interventional CT with tracked needles to facilitate lumbar facet needle guidance 4, while Galiano et al have shown that ultrasound (US) can be used instead of fluoroscopy or CT for lumbar facet joint injections [5]. US technology is both ubiquitous and considerably less expensive than fluoroscopy or CT, and eliminates radiation dose to both patient and clinician. However, on its own US can be difficult to interpret and the high frequencies required to adequately visualize small needles will not always penetrate deeply enough to visualize the target facet joint.

By integrating 2D US with virtual representations of anatomical targets and the tracked therapy delivery needle, it is possible to greatly enhance the US information available to the clinician. The overall goal of the present work is to develop an anesthesia delivery system that allows the integration of virtual models derived from diagnostic imaging such as CT and tracked tools, all intended to enhance real time US data. Our specific goal in the present work is to assess the accuracy of augmented reality (AR) assistance for US guided lumbar spinal facet injections. We evaluate two information delivery strategies: a simple system representing only the tools being used (US and needle), and a more elaborate system that also incorporates a patient specific spine model for additional guidance.

\section{Materials and Methods}

Four anesthetists and four anesthesia residents evaluated three different guidance systems (see Figure 10 to place a tracked needle on target for facet therapy injections in a lumbar spine phantom:

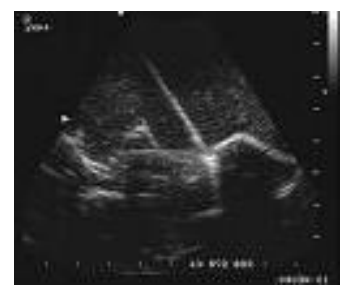

$G S_{u s}$

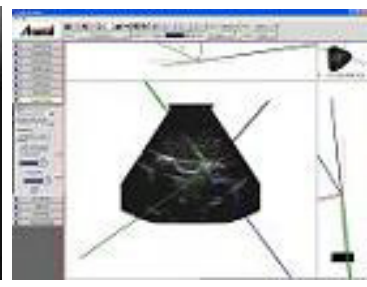

$G S_{\text {tools+ }}$

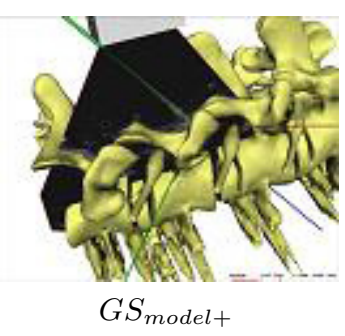

Fig. 1. The three guidance systems evaluated. The virtual needle tool also has $x, y$ and $\mathrm{z}$ axis extensions with $10 \mathrm{~mm}$ markers to help locate the tip and its trajectory. 
- US only $\left(G S_{u s}\right)$ : the users were restricted to using the US machine monitor. They were free to manipulate the location and angle for the US transducer;

- US plus virtual tool representations $\left(G S_{t o o l s+}\right)$ : the users had full use of ultrasound, plus representations of the US and needle tool: the AR display consisted of the normal fixed view of the US fan with 'top' and 'side' views showing the angle and tip of the needle relative to the US beam;

- Complete 3D AR system $\left(G S_{\text {model+ }}\right)$ : the users had full use of the US, representations of US transducer and needle, plus an anatomical model of the spine based on a high resolution CT. The user had full control of their view of the scene.

The $G S_{\text {tools }}$ system is intended for applications where no anatomical models are available (e.g., no pre-procedure imaging is done, as is the case for most peripheral nerve blocks). It was expected this system would have an advantage over US guidance since it allows the anesthetist to localize the needle tip even when it is not entirely in plane in the US. This system will be evaluated specifically for use in peripheral nerve block applications in future studies.

To date we have performed a single cadaver study to assess the logistics of translating our techniques to a clinical environment.

Apparatus. Our phantom experiments were carried out using a plastic lumbar spine that was rigidly fixed inside a lexan box using four high grade stainless steel screws to hold each vertebra (see Figure 2). A total of 17 concave landmark divots were machined randomly into the outside of the container for registration purposes. The spine was embedded in a tissue mimicking polyvinyl alcohol cryogel (PVA-C) material (10\% PVA, two freeze-thaw cycles) [6]. To remove needle tracks left behind in the phantom after repeated experiments, the PVA-C was heated above $60^{\circ}$ Celcius to melt it into a liquid state after which the freeze-thaw cycles were repeated to return the PVA to its solid tissue-mimicking state. The phantom was imaged using a 64 slice LightSpeed VCT scanner (General Electric, Milwaukee, WI, USA), $(0.441 \mathrm{~mm}$ by $0.441 \mathrm{~mm}$ in plane, $0.625 \mathrm{~mm}$ slice thickness), to generate an accurate surface model of the spine. An expert identified the ideal target facet locations in the phantom CT by manually segmenting each facet joint using ITK-SNAP (www.itksnap.org), and a spine model was generated using the Marching Cubes algorithm in VTK (Kitware Inc.), as shown in Figure 2. Landmark divot locations were defined in the CT image to register the tracked US and needle into the $\mathrm{CT} /$ model/target coordinate frame. Imaging was provided by an Aloka US system with a $5 \mathrm{MHz}$ neuro transducer.

Measuring Accuracy. For the actual therapy delivery, a magnetic tracking system is required since the delivery needle is capable of bending inside tissue. The Aurora MTS (NDI, Waterloo, ON, Canada) was used to track needles and US. The needle (stylus and cannula) used was a 5 degrees of freedom (DOF) 18 gauge, $200 \mathrm{~mm}$ long needle (Figure 2) with the sensor located at its tip. 6DOF sensors were used to track the US transducer and to provide a reference for the phantom itself. The US transducer was calibrated using a Z-bar phantom 


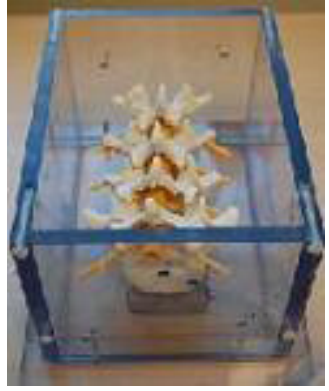

(a)

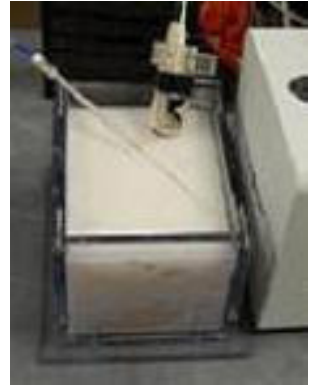

(b)

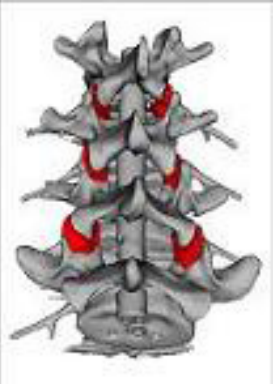

(c)

Fig. 2. Phantom, tools and targets: (a) spine inside the phantom, (b) phantom with tissue-mimicking PVA-C, tracked needle tool, US transducer and tracker field generator (on right), (c) Facet targets in context of the spine model generated from CT data

[7. Since the MTS has an inherent coordinate frame based on the magnetic field generator, all tool coordinate vectors $\left(\bar{p}_{f g}\right)$ are relative to this frame of reference. This could cause problems if the phantom or field generator move relative to each other during an experiment. Consequently the reference tool was rigidly attached to the spine phantom box to yield coordinates relative to the phantom, thus ensuring tracked tool coordinates $\left(\bar{p}_{m t s}\right)$ remained consistent with the phantom coordinate frame:

$$
\bar{p}_{m t s}=T_{r e f}^{-1}\left(\bar{p}_{f g}\right),
$$

where $T_{\text {ref }}$ represents the transform of the reference tool. In clinical applications, placing this reference tool near the target will also help compensate for patient shifting and breathing motion. The tracking coordinate frame was registered to the CT-generated model using a tracked sphere-tipped tool that mated with the divots on the outside of the phantom box, generating a very accurate pointbased registration. Using this point based registration transform $\left(T_{\text {phantom }}\right)$, we translated the measured needle coordinates $\left(\bar{p}_{m t s}\right)$ into the CT coordinate frame:

$$
\bar{p}_{\text {meas }}=T_{\text {phantom }}\left(\bar{p}_{m t s}\right)
$$

where $\bar{p}_{\text {meas }}$ is the measured needle tool tip location CT space. Trueness is then the distance defined by the magnitude of the error vector,

$$
d_{\text {err }}=\left|\bar{p}_{\text {error }}\right|=\left|\bar{p}_{\text {meas }}-\bar{p}_{\text {target }}\right| \text {, }
$$

where $\bar{p}_{\text {target }}$ is the closest point on the facet joint polydata as defined in the CT. When $\bar{p}_{\text {meas }}$ was located inside the target polydata, the error was zero.

Software. The $G S_{\text {model+ }}$ system used a software platform that presents the real-time tracked US and tools in a $3 \mathrm{D}$ context, allowing the user to interactively select which data to display: the pre-procedural CT, real time US image, in addition to geometric models, from any desired point of view 8 . 
With the $G S_{\text {model+ }}$ system, the user was able to toggle between two "standard views" of their own choosing. Typically, this feature allowed the user to flip between orthogonal views to provide accurate perception of needle trajectory and needle depth. The $G S_{\text {tools }}+$ system was based on the same software platform.

Implementation. Each subject gained familiarity with the AR guidance systems using a training apparatus consisting of tracked ultrasound and needle and a series of small targets in a water bath. Surgical targets were shown to the subjects prior to performing the experiment but were not available during the experiment, since these data would not be available during an actual needle therapy delivery. After the subjects were comfortable with the systems, they proceeded to place the delivery needle at the target locations. A total of six target locations were used: facet joints between L5 and L4, L4 and L3, L3 and L2. Each guidance modality was used on each target, yielding 18 deliveries for each subject, six for each guidance system. The target order and modality were uniquely randomized for each subject in order to minimize any bias.

Cadaver Study. A proof of concept cadaver study was performed using the $G S_{\text {model+ }}$ guidance system to assess the system in a clinical setting. This study was performed according to the University of Western Ontario requirements for the use of cadavers. Seven homologous points representing the spinal and lateral processes in the region of interest were defined in the pre-procedural CT and interactively in the OR using 2D US to perform the point based registration. This procedure took approximately five minutes. To properly mimic a clinical therapy, radio opaque dye was injected when the user believed the needle was in the correct position. An anesthesia resident performed the needle delivery using the $G S_{\text {model }}$ guidance system, placing radio opaque dye at the left and right L2-3 and L3-4 facet joints. Accuracy was assessed independently by a radiologist using a post-procedure CT (see Figure 3).

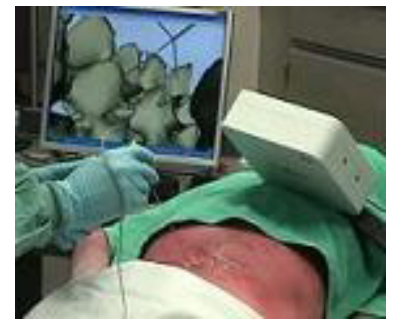

(a)

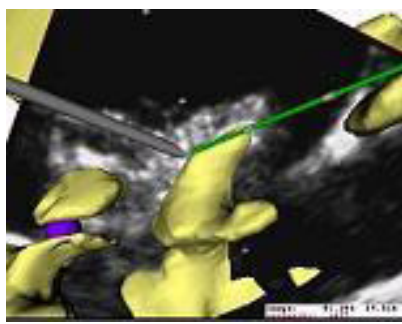

(b)

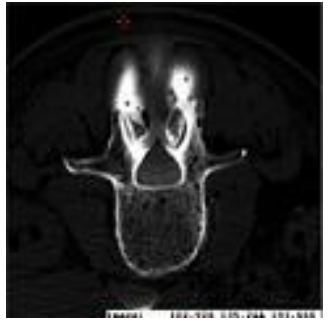

(c)

Fig. 3. Cadaver study and results. (a) OR environment showing AR scene, delivery needle and MTS field generator, (b) close-up of $G S_{\text {model+ }}$ system including needle (gray), US fan and projected $y$ axis (green), (c) post-procedure CT showing radio opaque dye at the L2-3 facet joints. 
Table 1. RMS distance errors in mm for each image guidance system. 'Correct target' excludes outliers where the subject identified the wrong facet joint but accurately reached the (incorrect) target.

\begin{tabular}{|l|r|c|c|}
\hline & $G S_{u s}$ & $G S_{\text {tools }}$ & $G S_{\text {model+ }}$ \\
\hline \hline all data & 10.22 & 8.45 & 0.57 \\
\hline correct target only & 5.77 & 4.87 & 0.57 \\
\hline
\end{tabular}

\section{Results and Discussion}

The phantom experiment was a $3 \times 6 \times 8$ factorial design, the factors being guidance system, target and user, respectively. An analysis of variance (ANOVA) was performed to test for significant variance across the three factors. When a significant difference was found in the ANOVA, Tukey's Honestly Significant Difference test was applied to determine which levels of the given factor were different. No significant difference was noted across users or targets $(p>0.05)$.

The use of $G S_{\text {model+ }}$ showed a marked superiority to $G S_{U S}$ and $G S_{\text {tools }}$. One curious phenomena was noted in several subjects: on six occasions using the $G S_{\text {tools }}$ system and five occasions with the $G S_{U S}$ system, users managed to correctly place the needle tip inside a facet joint, but selected the incorrect joint. This problem never arose with the $G S_{\text {model }}$ system. Table 1 displays root mean squared (RMS) distance error data considering all trials, including cases where the anesthetist placed the needle tip "correctly" in the wrong facet joint, as well as the distance error data excluding these cases. In both cases, there is a significant difference $(p<<0.05)$ between the $G S_{\text {model+ }}$ system and the other two systems. How common this form of error is in practice is unclear, however the use of an AR system incorporating anatomical models would appear to circumvent the problem.

Figure 4 provides a graphical representation of therapy delivery locations relative to targets. Overall, the $G S_{\text {tools }}+$ system showed slightly better RMS error than US alone, but not at a statistically significant level. The cognitive demands of this new guidance system may have contributed to these results, as several clinicians commented that the computer monitor was rather "busy". Larger sample sizes are planned for future research which should help to quantify the advantage of the $G S_{\text {tools }}$ system, particularly for peripheral nerve block applications where no pre-procedural imaging is done.

An unpaired t-test was performed to compare residents and anesthetist accuracy, and no significant difference was found. The anesthesia residents involved in this study had significant experience with US guided peripheral nerve blocks, so this lack of difference between novices and experts is not surprising. Only two of the participants had prior experience with facet joint injections. Several of the participants commented on the training and educational potential of the guidance systems, both for identifying anatomical landmarks (using the $G S_{\text {model }}$ system) and recognizing when the needle tip is in or out of the US plane ( $G S_{\text {tools }}$ system). Anecdotally, all users described the $G S_{\text {model+ }}$ 


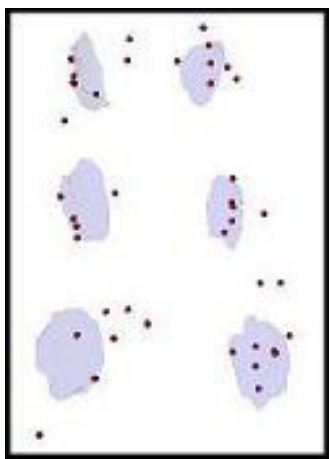

(a)

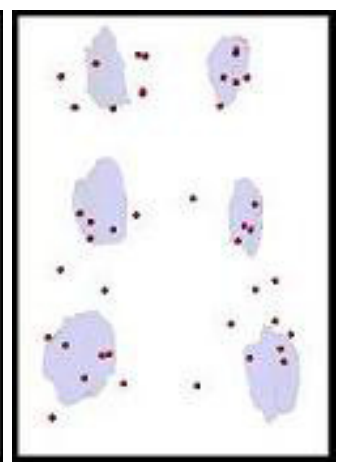

(b)

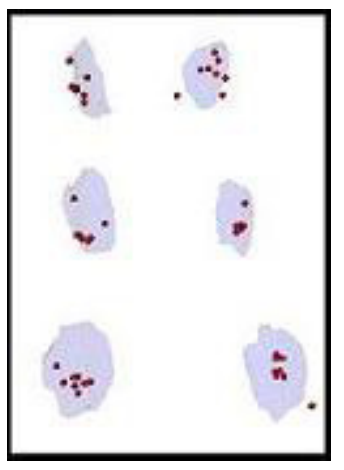

(c)

Fig. 4. Distribution of needle placements in coronal view: blue clouds are the facet joint targets, spheres are needle delivery locations. (a) US alone, (b) $G S_{\text {tools }}+$ system, (c) $G S_{\text {model+ }}$ system.

guidance system as a substantial improvement over existing systems (US alone and fluoroscopy).

Introducing the tracking system into the OR environment for the cadaver study posed no significant difficulties. Attention was paid to minimize the presence of magnetic materials in the vicinity of the MTS field generator. Although we did not record the number of needle penetrations necessary for accurate placement, the clinician commented that the new system greatly reduced the number of penetrations and the time required to position the needle correctly. The $x, y$, and $z$ axes projected from the virtual needle tip were especially useful for determining trajectory and tip location. The radiologist report stated signal enhancement (due to injected radio opaque dye) in and surrounding facet joints right and left, L2-3 and L3-4. This correlates with a clinically significant injection (see Figure 3 (c)).

\section{Conclusions and Future Work}

Our results indicate that AR assisted ultrasound guidance for facet injections shows tremendous promise for increasing patient safety and comfort, greatly reducing or even eliminating radiation dose to both patient and clinicians, as well as reducing health care costs. The use of tracked US and needles in an AR environment also has a great deal of potential for application in nerve block clinics and anesthesia training.

Our next steps include the application and assessment of several features, including a more clinically relevant CT to US registration algorithm. We are also evaluating the use of a volume rendered display of $3 \mathrm{D}$ reconstructed ultrasound data, since this would eliminate the need for registering pre-procedural CT data and models. The need to compensate for respiratory motion and changes in position during the clinical intervention may be reduced by the use of a tracked 
reference tool in the vicinity of the target tissues, though this will need further study. An assessment of the cognitive demands presented by the AR systems will also help to properly interpret their significance. Educational and clinical applications for peripheral nerve blocks are also in the planning stages.

Acknowledgments. We would like to thank the Department of Anesthesia, London Health Sciences Center, Canada for consultations and assistance with experiments. We would also like to thank Dr. Gord Campbell (National Research Council, London Canada) for assistance with PVA-C procedures and resources, Dr. Tim Wilson and Mahar Sabalbal (University of Western Ontario, London Canada) for assistance with geometric models used in the cadaver study, and CT technologists at LHSC- University Hospital, London, Ontario for CT data acquisition.

\section{References}

1. Boswell, M., Colson, J., Sehgal, N., Dunbar, E., Epter, R.: A systematic review of therapeutic facet joint interventions in chronic spinal pain. Pain Physician 10, 229-253 (2007)

2. Proschek, D., Kafchitsas, K., Rauschmann, M., Kurth, A., Vogl, T., Geiger, F.: Reduction of radiation dose during facet joint injection using the new image guidance system sabersource: a prospective study in 60 patients. European spine journal 18, 546-553 (2009)

3. Wood, B., Zhang, H., Durrani, A., Glossop, N., Ranjan, S., Lindisch, D., Levy, E., Banovac, F., Borgert, J., Krueger, S., Kruecker, J., Viswanathan, A., Cleary, K.: Navigation with electromagnetic tracking for interventional radiology procedures:A feasibility study. Journal of vascular and interventional radiology 16, 493-505 (2005)

4. Bruners, P., Penzkofer, T., Nagel, M., Elfring, R., Gronloh, N., Schmitz-Rode, T., Gunther, R., Mahnken, A.: Electromagnetic tracking for CT-guided spine interventions: phantom, ex-vivo and in-vivo results. European Radiology 19, 990-994 (2009)

5. Galiano, K., Obwegeser, A.A., Walch, C., Schatzer, R., Ploner, F., Gruber, H.: Ultrasound-guided versus computed tomography-controlled facet joint injections in the lumbar spine: A prospective randomized clinical trial. Regional Anesthesia and Pain Medicine 32(4), 317-322 (2007)

6. Surry, K., Austin, H., Fenster, A., Peters, T.: Poly(vinyl alcohol) cryogel phantoms for use in ultrasound and MR imaging. Physics in Medicine and Biology 49(24), 5529-5546 (2004)

7. Gobbi, D., Comeau, R., Peters, T.: Ultrasound probe tracking for real-time ultrasound/MRI overlay and visualization of brain shift. In: Taylor, C., Colchester, A. (eds.) MICCAI 1999. LNCS, vol. 1679, pp. 920-927. Springer, Heidelberg (1999)

8. Moore, J., Guiraudon, G., Jones, D., Hill, N., Wiles, A., Bainbridge, D., Wedlake, C., Peters, T.: 2D ultrasound augmented by virtual tools for guidance of interventional procedures. Studies in Health Technology and Informatics, 322-327 (2007) 